\title{
Formation of Fe Cluster Superlattice in a Metal-Organic Quantum-Box Network
}

\author{
Marina Pivetta, ${ }^{1}$ Giulia E. Pacchioni, ${ }^{1}$ Uta Schlickum, ${ }^{1,2}$ Johannes V. Barth ${ }^{3}$ and Harald Brune ${ }^{1}$ \\ ${ }^{1}$ Institute of Condensed Matter Physics, Ecole Polytechnique Fédérale de Lausanne (EPFL), CH-1015 Lausanne, Switzerland \\ ${ }^{2}$ Max-Planck-Institut für Festkörperforschung, D-70569 Stuttgart, Germany \\ ${ }^{3}$ Physik Department E20, Technische Universität München, D-85748 Garching, Germany \\ (Received 4 October 2012; revised manuscript received 20 December 2012; published 19 February 2013)
}

\begin{abstract}
We report on the self-assembly of $\mathrm{Fe}$ adatoms on a $\mathrm{Cu}(111)$ surface that is patterned by a metal-organic honeycomb network, formed by coordination of dicarbonitrile pentaphenyl molecules with $\mathrm{Cu}$ adatoms. $\mathrm{Fe}$ atoms landing on the metal surface are mobile and steered by the quantum confinement of the surface state electrons towards the center of the network hexagonal cavities. In cavities hosting more than one $\mathrm{Fe}$, preferential interatomic distances are observed. The adatoms in each hexagon aggregate into a single cluster upon gentle annealing. These clusters are again centered in the cavities and their size is discerned by their distinct apparent heights.
\end{abstract}

DOI: 10.1103/PhysRevLett.110.086102

PACS numbers: 68.37.Ef, 81.16.Dn

Self-assembly of adatoms or small clusters at surfaces enables the bottom-up fabrication of well-defined nanoscale structures. Well ordered nanostructure superlattices can be created by the nucleation and growth on template surfaces exhibiting long period adatom binding energy variations, such as equidistant pinning sites [1] or networks of repulsive line defects [2]. Besides surface reconstructions and stress relief patterns of epitaxial thin films, nanoporous metalorganic networks [3,4] are potential candidates for such templates. So far, deposition of metal atoms on the latter systems resulted in the decoration of the organic molecules themselves or of the coordination nodes, but not in equidistant clusters on the substrate [5].

An additional source of order can be introduced by the quasi-two-dimensional (2D) electron gas of a surface state mediating long-range adsorbate interactions [6-8]. On homogeneous surfaces, they stabilize atomic superlattices [6-9]. Surface state confinement by static scatterers results in local density of state (LDOS) patterns that influence the adsorbate binding energy. In 1D structures formed by substrate steps or strings of atoms or molecules, this leads to $1 \mathrm{D}$ confinement of adsorbed atoms [10-13]. The surface state LDOS patterns formed in a network of hexagonal molecular cavities have been demonstrated to influence the binding sites of adsorbed CO molecules [14].

Here, we demonstrate strong surface state confinement by a metal-organic network, preferred adatom locations due to the LDOS pattern created in each cavity, and aggregation of these atoms to a single cluster per network cavity, thus giving rise to a cluster superlattice with the period of the metal-organic template. Our system is a honeycomb network with $\approx 5 \mathrm{~nm}$ period formed by dicarbonitrile pentaphenyl $\left(\mathrm{NC}-\mathrm{Ph}_{5}-\mathrm{CN}\right)$ molecules and $\mathrm{Cu}$ atoms on $\mathrm{Cu}(111)$, and the steered adatoms are $\mathrm{Fe}$.

The $\mathrm{Cu}(111)$ substrate has been prepared by $\mathrm{Ar}^{+}$sputter and annealing cycles. The $\mathrm{NC}-\mathrm{Ph}_{5}-\mathrm{CN}$ molecules [3] were evaporated from a molecular effusion cell at $230^{\circ} \mathrm{C}$.
The honeycomb lattice is formed by threefold coordination via the $\mathrm{CN}$ groups to $\mathrm{Cu}$ adatoms [15-17] present on the substrate terraces as dilute $2 \mathrm{D}$ gas at the substrate temperature during deposition of $250 \mathrm{~K}$. Fe has been evaporated from an $e$-beam evaporator with the sample placed in the low temperature scanning tunneling microscope (STM) [18] and held at $T_{\text {dep }} \approx 12 \mathrm{~K}$. The Fe coverage $\Theta$ is expressed in monolayers (ML), 1 ML corresponding to one Fe adatom per substrate atom. It has been calibrated by counting isolated $\mathrm{Fe}$ atoms resulting from deposition of small amounts onto the bare $\mathrm{Cu}(111)$ surface. All measurements have been carried out at $5 \mathrm{~K}$.

Figure 1(a) shows a sketch of $\mathrm{NC}-\mathrm{Ph}_{5}-\mathrm{CN}$ that has a geometric length of $2.53 \mathrm{~nm}$ [3]. The $\left(\mathrm{NC}-\mathrm{Ph}_{5}-\mathrm{CN}\right)_{3} \mathrm{Cu}_{2}$ network is shown in Fig. 1(b). Figure 1(c) presents a closeup view together with the proposed model. The molecules are oriented along $\langle 11 \overline{2}\rangle$, with the $\mathrm{Cu}$ coordination atoms occupying fcc hollow sites [19]. The network unit cell is described by a

$$
\left(\begin{array}{cc}
19 & 1 \\
-1 & 20
\end{array}\right)
$$

matrix with respect to the $\mathrm{Cu}(111)$ primitive vectors; it covers 381 substrate unit cells. This model yields a periodicity of $4.99 \mathrm{~nm}$, in agreement with the experimental value of $4.97 \pm 0.04 \mathrm{~nm}$. Coordination to metal adatoms is inferred (i) from the fact that deposition at lower temperatures, i.e., in the absence of the 2D gas of $\mathrm{Cu}$ atoms $[16,17]$, leads to the formation of other phases [20] and (ii) from the similarity to the $\left(\mathrm{NC}-\mathrm{Ph}_{n}-\mathrm{CN}\right)_{3} \mathrm{Co}_{2}$ structures, $n \in$ $\{3,4,5,6\}$, formed on $\operatorname{Ag}(111)[3,4]$. Close inspection of Fig. 1(c) reveals that in every other of the threefold coordination nodes, the molecules do not point straight towards the coordination atom, implying network chirality [21]. Therefore, the cavities are quasihexagons with alternating short and long sides and possess threefold symmetry. 


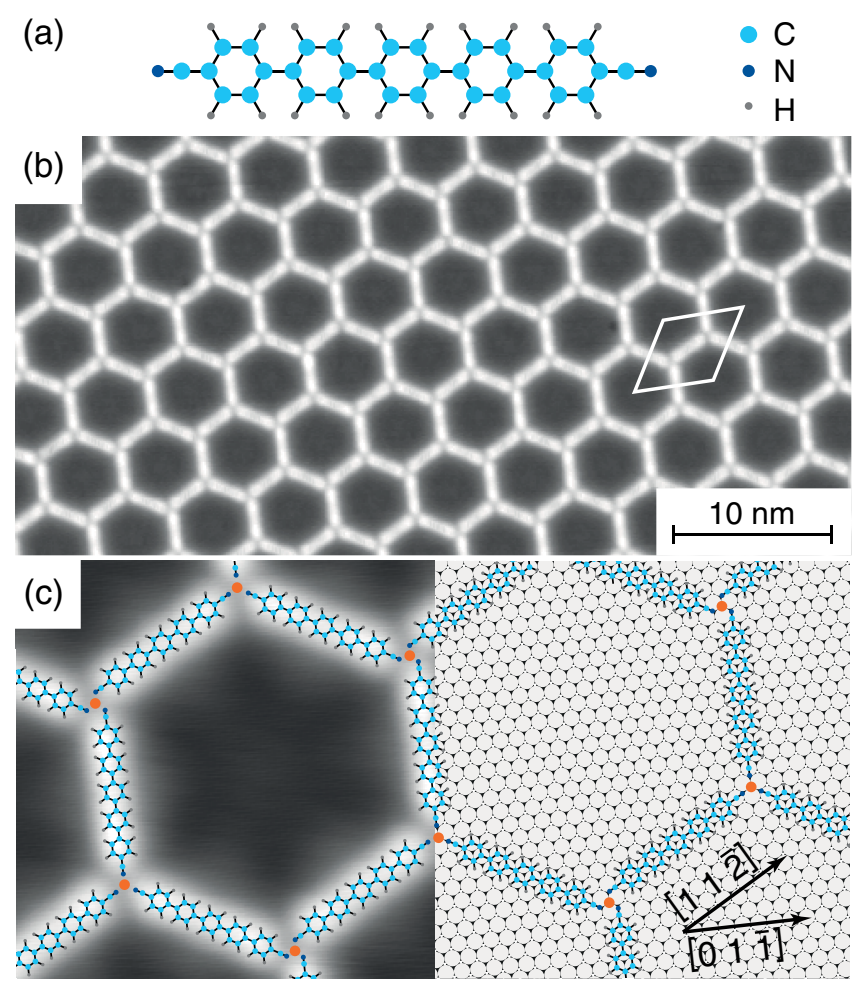

FIG. 1 (color online). (a) Structure of $\mathrm{NC}-\mathrm{Ph}_{5}-\mathrm{CN}$. (b) STM image showing a $\mathrm{Cu}(111)$ terrace covered by the $\left(\mathrm{NC}-\mathrm{Ph}_{5}-\mathrm{CN}\right)_{3} \mathrm{Cu}_{2}$ honeycomb lattice $\left(V_{t}=-0.6 \mathrm{~V}\right.$, $\left.I_{t}=20 \mathrm{pA}\right)$. (c) Close-up view $\left(V_{t}=-0.02 \mathrm{~V}, I_{t}=20 \mathrm{pA}\right)$ and corresponding model.

The confinement of surface state electrons in the cavities is evident from the $d I / d V$ data shown in Fig. 2. The spectra show strong spatial dependence and the $d I / d V$ maps reveal the LDOS patterns of the confined states [22,23]. The cavity geometry is close to the one of adatom and vacancy islands of the (111) surfaces of $\mathrm{Ag}$ [24] and $\mathrm{Cu}$ [25], but in the present case, confinement is accomplished by the metal-organic network [26]. We can clearly discern five eigenstates identified and labeled as state $1,2,4,7$, and 10 [24]. The complexity of their spatial distribution increases with energy, as demonstrated by the $d I / d V$ maps of Fig. 2(b). Notice that the threefold symmetry of the cavity, originating from the two types of coordination nodes, is also reflected in the DOS pattern as especially visible for higher order states, such as 7 and 10. No quantum state coincides with the Fermi level $E_{\mathrm{F}}$. However, state 4 at $-70 \mathrm{mV}$ has high intensity at the cavity center and thus it contributes to the LDOS maximum at the center of the hexagon at $E_{\mathrm{F}}$.

Deposition of minute amounts of Fe onto the metalorganic network leads to isolated Fe atoms in the cavities, coexisting with atoms adsorbed on the polyphenyl backbones, see Fig. 3(a). Considering the size of the network unit cell, one would expect $1.33 \mathrm{Fe}$ atoms per hexagon at the chosen Fe coverage. From a statistical analysis carried out on more than thousand cavities, we find that there are

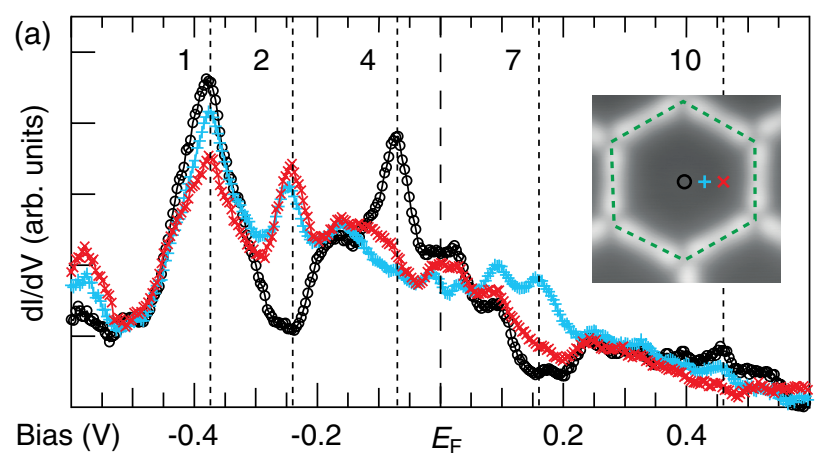

(b)

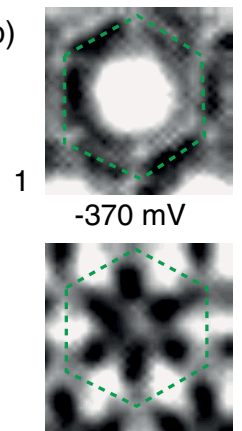

$E_{\mathrm{F}}$

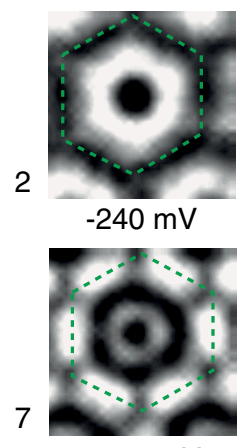

$+160 \mathrm{mV}$

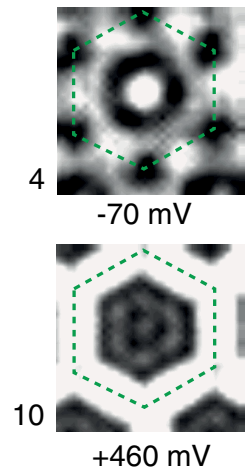

FIG. 2 (color online). (a) $d I / d V$ spectra acquired at the locations in the hexagonal cavity indicated in the inset. (b) $d I / d V$ maps at the indicated voltages extracted from an array of $32 \times 32$ open feedback loop point spectra. (setpoint: $V=-0.6 \mathrm{~V}, I=0.4 \mathrm{nA}$; lock-in acquisition, $V_{\text {mod }}=10 \mathrm{mV}$ peak-to-peak at $437 \mathrm{~Hz}$ ).

only $1.03 \pm 0.03$ atoms. This number is expected if we remove the sites occupied by network components $(23 \%)$, leading to an inner cavity size of 293 sites. This result indicates that atoms landing on linkers and nodes do not diffuse to the pores. Statistical analysis yields the occupancy histogram presented in Fig. 3(b). The black dots show the filling expected from a binomial distribution

$$
P(k, n, \Theta)=\left(\begin{array}{l}
n \\
k
\end{array}\right) \Theta^{k}(1-\Theta)^{n-k}
$$

where $\Theta$ is the adatom coverage, $n$ the inner cavity size expressed in substrate unit cells, and $k \in\{0,1, \ldots, 6\}$ the occupancy [2]. There is excellent agreement between experiment and binomial statistics indicating that those adatoms landing on the substrate are trapped in the cavities. Notice that in this very low coverage limit, the occupancy is well described also by a Poisson distribution.

Figure 3(a) further reveals that the Fe atoms in pores with an occupancy $k \geq 2$ have preferred nearest neighbor distances and thus, for $k \geq 3$, they form small patches of a superlattice. The origin of this ordering is the long-range oscillatory interaction mediated by the surface state electrons. The period of the interaction energy $E_{\text {int }}$ is given by half the Fermi wavelength of the surface state, the adatom nearest neighbor distance $d_{\mathrm{nn}}$ is the position of the first 

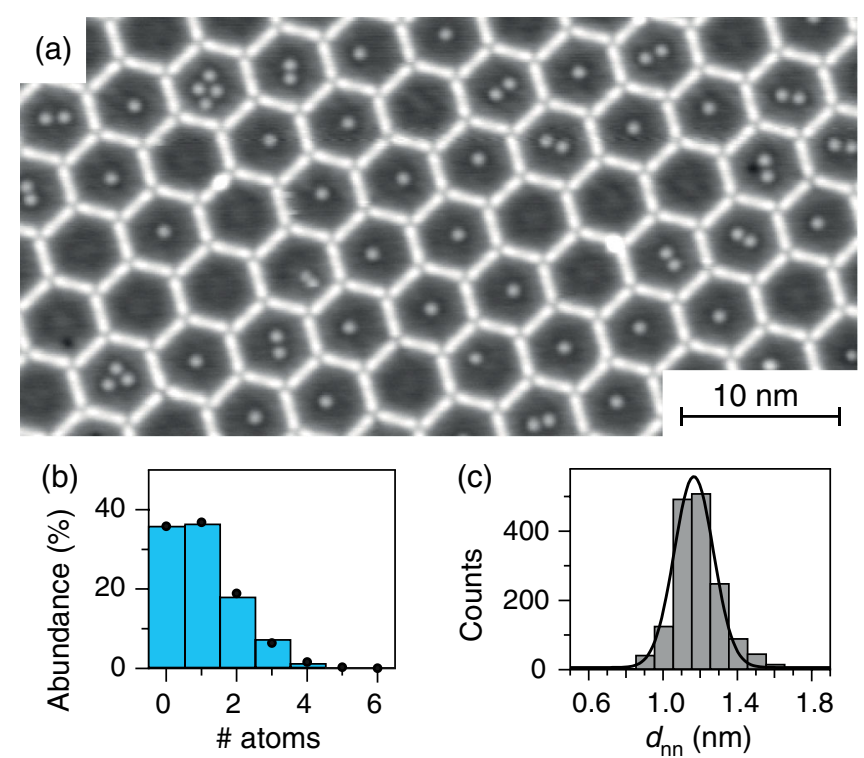

FIG. 3 (color online). (a) STM image showing individual $\mathrm{Fe}$ adatoms in the template cavities $\left(\Theta=3.5 \times 10^{-3} \mathrm{ML}\right.$, $V_{t}=-0.2 \mathrm{~V}, I_{t}=20 \mathrm{pA}$ ). (b) Measured occupancy histogram (bars) and calculated binomial distribution (dots). (c) Nearestneighbor distance histogram for cavity occupation above one.

minimum of $E_{\text {int }}$ given by the adatom scattering phase [6,7] and the overlap with the short range repulsion inhibiting cluster formation $[8,27]$. The formation of these patches at $T_{\text {dep }} \approx 12 \mathrm{~K}$ is consistent with the reported values of diffusion [28-30] and short range repulsive [29] barriers for $\mathrm{Fe}$ adatoms on $\mathrm{Cu}(111)$. The $d_{\mathrm{nn}}$ histogram shown in Fig. 3(c) has been obtained from analyzing 1600 adatom pairs. The deduced mean distance $d_{\mathrm{nn}}=1.1 \pm 0.1 \mathrm{~nm}$ is in agreement with the values of $1.2 \mathrm{~nm} \mathrm{[29]} \mathrm{and} 1.1 \mathrm{~nm} \mathrm{[30]} \mathrm{reported} \mathrm{for}$ Fe on bare $\mathrm{Cu}(111)$. The distance distribution is significantly more narrow than the ones reported for adatom superlattices on homogeneous surfaces. This is experimental evidence for the predicted enhancement of the surface state mediated interaction by its confinement [31].

Further inspection of Fig. 3(a) reveals that the Fe atoms, as well as the center of mass of the dimers and trimers, are centered in the cavities and that the assemblies show preferred orientations. Figure 4 shows the Fe adsorption site statistics, once in 2D plots and once as normalized radial abundance, with respect to the quasihexagon center. The normalization compensates for the linearly increasing number of fcc adsorption sites [28] per bin with increasing radius. The results for single occupancy are shown in Fig. 4(a). Random distribution would give rise to the horizontal dashed line while the observed positions are strongly peaked in the cavity center. The inset displays the 2D plot where each black dot represents a measured Fe position. The dots are isotropically distributed in immediate vicinity of the center.

The results for cavities occupied by two Fe atoms are presented in Fig. 4(b). The radial histogram exhibits

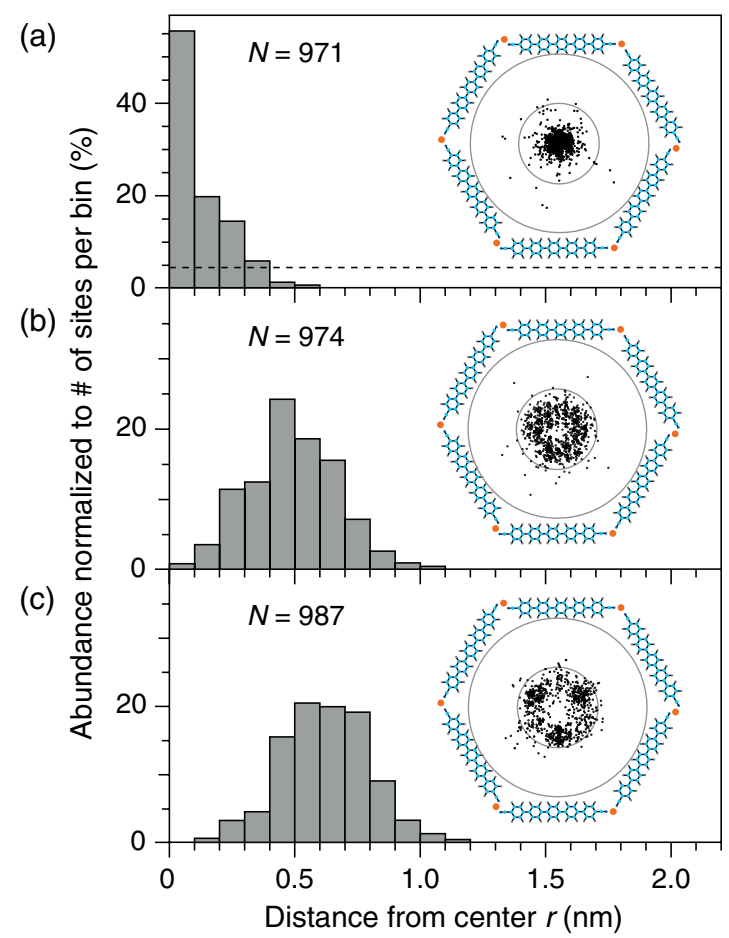

FIG. 4 (color online). Position of the Fe adatoms in the hexagonal cavities for occupancies of (a) one, (b) two, and (c) three Fe atoms. $N$ is the number of analyzed adatoms. Main figures: radial distribution histograms normalized to the number of adsorption sites for each bin. Insets: adatom position 2D plots (black dots) with circles at $r=1.0$ and $2.2 \mathrm{~nm}$.

a broad peak centered at $0.5 \mathrm{~nm}$. The adsorption site map indicates that the pairs occupy the center of the cavity while keeping their preferential distance of $1.1 \mathrm{~nm}$. In contrast to the first impression gained from Fig. 3(a), the dimers show no preferential azimuthal orientation. The radial distribution of cavities hosting three $\mathrm{Fe}$ atoms, see Fig. 4(c), shows a broad peak at $0.65 \mathrm{~nm}$ in agreement with the value expected for adatoms occupying the vertices of an equilateral triangle of $1.1 \mathrm{~nm}$ side centered in the cavity. Moreover, the two-dimensional map reveals a preferential azimuthal orientation with threefold symmetry.

Spatial maxima of the surface state LDOS at $E_{\mathrm{F}}$ were found to be the preferred adsorption locations for Co [32] and $\mathrm{Cu}$ [12] adatoms on $\mathrm{Cu}(111)$. Since $\mathrm{Co}, \mathrm{Cu}$, and also $\mathrm{Fe}$ adatoms present comparable (1.1 to $1.3 \mathrm{~nm}$ ) nearestneighbor distances $[7,8,29,30]$, their interaction potentials and scattering properties are similar and we attribute the steering of $\mathrm{Fe}$ adatoms towards the cavity center to the LDOS at $E_{\mathrm{F}}$ having a maximum there. For cavities hosting more than one adatom, the superposition of two attractive potentials has to be considered, one arising from the confinement due to the molecules, the other created by the adatoms themselves $[11,13]$. The adatom position distribution observed in Fig. 4(c) reflects the threefold symmetry of the cavity. The preferred triangle orientation is such that the 

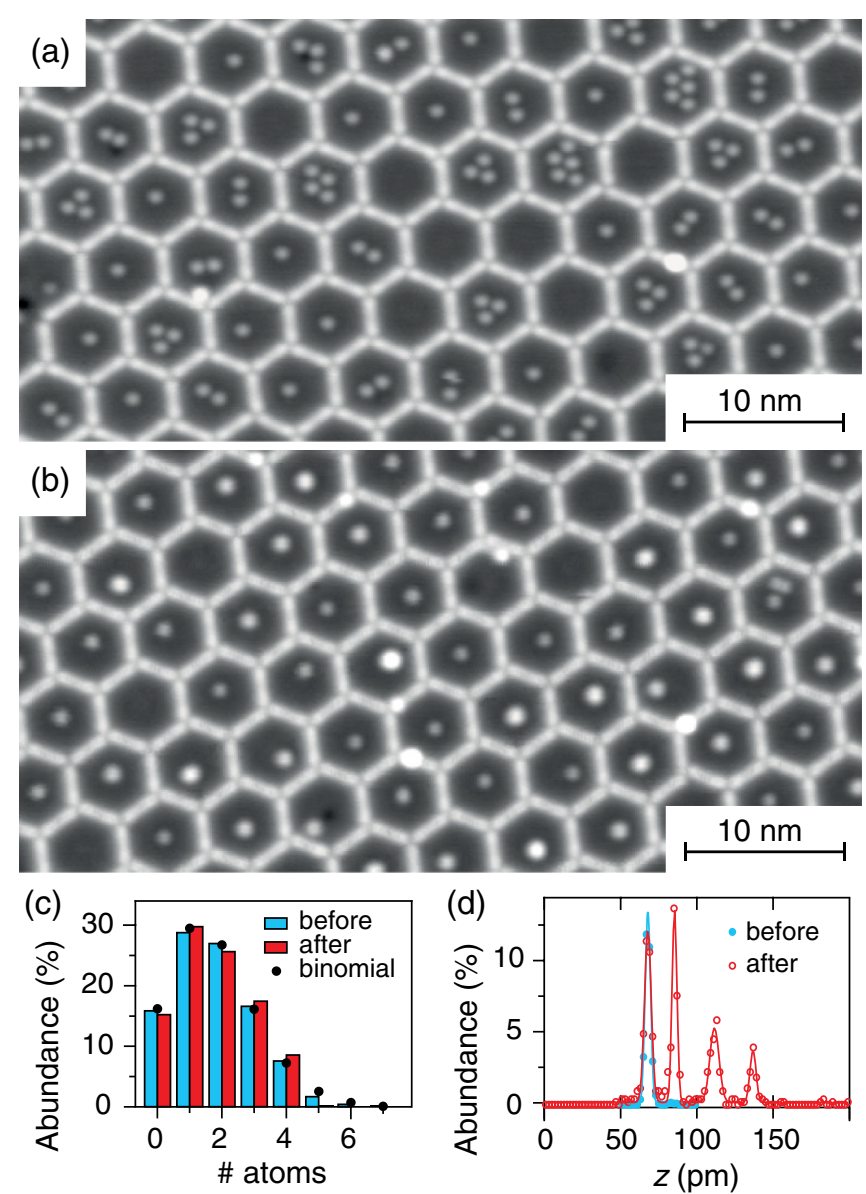

(e)

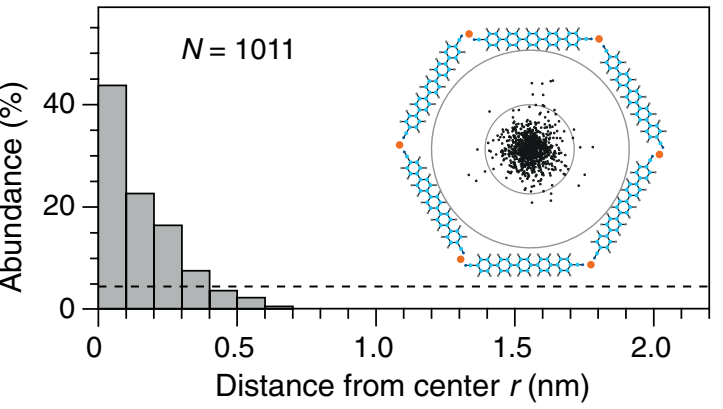

FIG. 5 (color online). STM images of Fe on template before (a) and after (b) annealing. Two different regions of the sample are imaged $\left(\Theta=7.0 \times 10^{-3} \mathrm{ML}, V_{t}=-0.5 \mathrm{~V}, I_{t}=50 \mathrm{pA}\right.$, $T_{\text {ann }}=18 \mathrm{~K}$ ). (c) Measured occupancy histogram before and after annealing (bars) compared with calculated binomial distribution (dots). (d) Apparent height histogram before and after annealing. (e) Positions of the Fe clusters in the cavity, as in Fig. 4.

adatoms are close to the short hexagon sides. The isotropic dimer distribution is rationalized by the incompatibility of twofold dimer and threefold cavity symmetry.

In the following, we demonstrate the creation of centered Fe clusters with known size by careful annealing of the sample prepared with pores containing isolated $\mathrm{Fe}$ atoms. Figure 5(a) shows the sample with an average of 2.06 atoms per cavity. The occupation histogram in Fig. 5(c), left (blue) bars, follows again a binomial distribution. Figure 5(b) shows the same sample after annealing to $18 \mathrm{~K}$. Almost all the occupied cells (97\%) host now a single object, while the proportion of empty pores is unchanged, see right (red) bars in Fig. 5(c). As for the adatoms before annealing, also the clusters are preferentially located at the center of the hexagon, as demonstrated in Fig. 5(e). Moreover, the number of adatoms or clusters adsorbed on the molecules has not changed significantly after annealing. In Fig. 5(d), we show the apparent height histogram of the $\mathrm{Fe}$ atoms before the annealing, for cavities with single occupancy, see filled (blue) dots. The apparent height distribution of the clusters obtained after annealing is displayed as open (red) circles. The number and apparent height of adatoms before and after annealing is identical; therefore, single occupancy cavities remained unchanged. Remarkably, additional well distinct peaks in the apparent height distribution are visible at 85, 112 , and $137 \mathrm{pm}$. They are identified as dimer, trimer, and tetramer clusters, respectively, as evidenced from the peak areas being in agreement with the cavity occupancy before annealing, see Fig. 5(c), right (red) bars. At the chosen annealing temperature, the thermal energy is enough for the Fe adatoms to overcome the repulsive barrier and to aggregate, consistently with the observations reported for $\mathrm{Fe}$ on bare $\mathrm{Cu}(111)$ [29,30]. However, the annealing temperature is insufficient for the adatoms to climb on the molecules or to diffuse to another cavity, indicating that there is a higher energy barrier for these processes.

In conclusion, we have shown that a two-dimensional metal-organic honeycomb network on $\mathrm{Cu}(111)$ acts as an efficient and versatile template for the self-assembly of $\mathrm{Fe}$ atoms and of few-atom aggregates in the pore centers. The mechanism behind it is electron mediated mutual interaction between the adatoms reinforced by the quantum confinement of surface state electrons in the cavities. This approach is expected to be generally applicable for related nanoporous templates and enables the realization of welldefined cluster sizes and chemical environments.

We acknowledge M. Ruben and S. Klyatskaya for the synthesis of the molecules and the Swiss National Science Foundation for financial support.

[1] M. Schmid, G. Kresse, A. Buchsbaum, E. Napetschnig, S. Gritschneder, M. Reichling, and P. Varga, Phys. Rev. Lett. 99, 196104 (2007).

[2] H. Brune, M. Giovannini, K. Bromann, and K. Kern, Nature (London) 394, 451 (1998).

[3] U. Schlickum, R. Decker, F. Klappenberger, G. Zoppellaro, S. Klyatskaya, M. Ruben, I. Silanes, A. Arnau, K. Kern, H. Brune, and J. V. Barth, Nano Lett. 7, 3813 (2007).

[4] D. Kühne, F. Klappenberger, R. Decker, U. Schlickum, H. Brune, S. Klyatskaya, M. Ruben, and J. V. Barth, J. Am. Chem. Soc. 131, 3881 (2009). 
[5] R. Decker, U. Schlickum, F. Klappenberger, G. Zoppellaro, S. Klyatskaya, M. Ruben, J. V. Barth, and H. Brune, Appl. Phys. Lett. 93, 243102 (2008).

[6] P. Hyldgaard and M. Persson, J. Phys. Condens. Matter 12, L13 (2000).

[7] J. Repp, F. Moresco, G. Meyer, K. H. Rieder, P. Hyldgaard, and M. Persson, Phys. Rev. Lett. 85, 2981 (2000).

[8] N. Knorr, H. Brune, M. Epple, A. Hirstein, M. A. Schneider, and K. Kern, Phys. Rev. B 65, 115420 (2002).

[9] F. Silly, M. Pivetta, M. Ternes, F. Patthey, J. P. Pelz, and W. D. Schneider, Phys. Rev. Lett. 92, 016101 (2004).

[10] H.F. Ding, V.S. Stepanyuk, P.A. Ignatiev, N. N. Negulyaev, L. Niebergall, M. Wasniowska, C. L. Gao, P. Bruno, and J. Kirschner, Phys. Rev. B 76, 033409 (2007).

[11] Y. Pennec, W. Auwärter, A. Schiffrin, A. Weber-Bargioni, A. Riemann, and J. V. Barth, Nat. Nanotechnol. 2, 99 (2007).

[12] N. N. Negulyaev, V. S. Stepanyuk, L. Niebergall, P. Bruno, W. Hergert, J. Repp, K.-H. Rieder, and G. Meyer, Phys. Rev. Lett. 101, 226601 (2008).

[13] A. Schiffrin, J. Reichert, W. Auwärter, G. Jahnz, Y. Pennec, A. Weber-Bargioni, V.S. Stepanyuk, L. Niebergall, P. Bruno, and J. V. Barth, Phys. Rev. B 78, 035424 (2008).

[14] Z. Cheng, J. Wyrick, M. Luo, D. Sun, D. Kim, Y. Zhu, W. Lu, K. Kim, T. L. Einstein, and L. Bartels, Phys. Rev. Lett. 105, 066104 (2010).

[15] S. L. Tait, A. Langner, N. Lin, S. Stepanow, C. Rajadurai, M. Ruben, and K. Kern, J. Phys. Chem. C 111, 10982 (2007).

[16] G. Pawin, K. L. Wong, D. Kim, D. Sun, L. Bartels, S. Hong, T. S. Rahman, R. Carp, and M. Marsella, Angew. Chem., Int. Ed. Engl. 47, 8442 (2008).

[17] M. Matena, M. Stöhr, T. Riehm, J. Björk, S. Martens, M. S. Dyer, M. Persson, J. Lobo-Checa, K. Müller, M. Enache, H. Wadepohl, J. Zegenhagen, T. A. Jung, and L. H. Gade, Chem. Eur. J. 16, 2079 (2010).
[18] R. Gaisch, J. K. Gimzewski, B. Reihl, R. R. Schlittler, M. Tschudy, and W. D. Schneider, Ultramicroscopy 42-44, 1621 (1992).

[19] J. Repp, G. Meyer, K.-H. Rieder, and P. Hyldgaard, Phys. Rev. Lett. 91, 206102 (2003).

[20] M. Pivetta, G. E. Pacchioni, U. Schlickum, and H. Brune (to be published).

[21] S. Stepanow, N. Lin, D. Payer, U. Schlickum, F. Klappenberger, G. Zoppellaro, M. Ruben, H. Brune, J. V. Barth, and K. Kern, Angew. Chem., Int. Ed. Engl. 46, 710 (2007).

[22] M. F. Crommie, C. P. Lutz, and D. M. Eigler, Science 262, 218 (1993).

[23] J. Kliewer, R. Berndt, and S. Crampin, New J. Phys. 3, 22 (2001).

[24] J. Li, W.-D. Schneider, S. Crampin, and R. Berndt, Surf. Sci. 422, 95 (1999).

[25] L. Niebergall, G. Rodary, H. F. Ding, D. Sander, V. S. Stepanyuk, P. Bruno, and J. Kirschner, Phys. Rev. B 74, 195436 (2006).

[26] F. Klappenberger, D. Kühne, W. Krenner, I. Silanes, A. Arnau, F. J. García de Abajo, S. Klyatskaya, M. Ruben, and J. V. Barth, Phys. Rev. Lett. 106, 026802 (2011).

[27] A. Bogicevic, S. Ovesson, P. Hyldgaard, B. I. Lundqvist, H. Brune, and D. R. Jennison, Phys. Rev. Lett. 85, 1910 (2000).

[28] Y. Mo, K. Varga, E. Kaxiras, and Z. Y. Zhang, Phys. Rev. Lett. 94, 155503 (2005).

[29] N. N. Negulyaev, V. S. Stepanyuk, L. Niebergall, P. Bruno, W. Auwärter, Y. Pennec, G. Jahnz, and J. V. Barth, Phys. Rev. B 79, 195411 (2009).

[30] X. P. Zhang, B. F. Miao, L. Sun, C. L. Gao, A. Hu, H. F. Ding, and J. Kirschner, Phys. Rev. B 81, 125438 (2010).

[31] V.S. Stepanyuk, N. N. Negulyaev, L. Niebergall, and P. Bruno, New J. Phys. 9, 388 (2007).

[32] V. S. Stepanyuk, A. N. Baranov, D. V. Tsivlin, W. Hergert, P. Bruno, N. Knorr, M. A. Schneider, and K. Kern, Phys. Rev. B 68, 205410 (2003). 